\title{
USANDO AS LENTES DA ESTRATÉGIA PARA COMPREENDER OS DETERMINANTES DO DESEMPENHO EM PROJETOS DE PESQUISA E INOVAÇÃO AGROPECUÁRIA
}

\section{Pedro Canna Brazil Ramos}

Mestre em Administração pela Universidade Federal da Bahia - UFBA com período sanduíche na University of Toronto, Canadá

pedro.brazil@embrapa.br (Brasil)

\section{Sandro Cabral}

Doutor em Administração pela Universidade Federal da Bahia - UFBA

Professor da Universidade Federal da Bahia - UFBA

scabral@ufba.br (Brasil)

\section{RESUMO}

O presente trabalho objetiva identificar os fatores que afetam o desempenho de projetos de pesquisa e inovação agropecuária numa instituição pública de pesquisa. Selecionou-se uma amostra de 40 projetos de pesquisa da Embrapa e os resultados apontam que equipes heterogêneas e a formação de parcerias, redes e alianças influenciam positivamente no número de tecnologias geradas pelos projetos de pesquisa. O trabalho apontou um trade-off entre número de publicações científicas dos projetos e o número de tecnologias geradas pelos projetos. O número de artigos científicos gerados está correlacionado com a homogeneidade da equipe e com número de pesquisadores envolvidos nos projetos.

Palavras-chave: Estratégia; Inovação em pesquisa agropecuária; Desempenho. 


\section{INTRODUÇÃO}

Alguns bens e serviços são marcados por dois tipos de externalidades. O primeiro tipo versa sobre os impactos sociais e ambientais e o segundo refere-se aos elevados riscos inerentes ao processo de inovação (Mazzucato, 2013). Embora os resultados positivos possam ser apropriados por parcelas amplas da população, organizações orientadas a lucro podem evitar investir nos estágios iniciais do processo de inovação, uma vez que atores a jusante podem capturar os esforços realizados sem necessariamente remunerar aqueles que incorreram nos custos correlatos (Roper, Vahter \& Love, 2013). Como forma de fomentar o desenvolvimento tecnológico, governos frequentemente assumem um papel ativo no processo de inovação (Rezaie et al, 2012), em que pesem os problemas de inflexibilidade governamental, baixa intensidade de incentivos no setor público e de dificuldades políticas para contemplar interesses focalizados (Williamson, 1999). Esse é o caso de atividades como vacinas para doenças negligenciadas, desenvolvimentos tecnológicos na área de agricultura para culturas do mundo em desenvolvimento e tecnologias limpas. Nessa seara, agentes governamentais que se propõem a ser agentes de inovação tecnológica, necessitam desenvolver um conjunto de estratégias no sentido de gerar, acumular e potencializar competências dinâmicas capazes de conferir o desempenho necessário para o atendimento do interesse público (Klein et al, 2013).

Avanços recentes na área de estratégia e inovação apontam para a necessidade de se compreender os aspectos correlacionados ao desempenho em tais atividades (Martinsuo \& Poskela, 2011; Teece, 2010; Terzioiski, 2010). Muito pouco se sabe sobre os aspectos subjacentes as relações entre competências, redes colaborativas inter-organizacionais e desempenho observado. Considerando a estratégia um padrão de escolhas e decisões organizacionais voltadas à obtenção de desempenho (Porter, 1996), o presente trabalho objetiva compreender os fatores que afetam o desempenho de projetos de pesquisa e inovação realizados em instituições públicas de pesquisa.

Para tanto, recorreu-se ao exame de projetos de pesquisa da Empresa Brasileira de Pesquisa Agropecuária (EMBRAPA) - Unidade Mandioca e Fruticultura - concluídos entre os anos de 2005 e 2012. A base de dados única utilizada nesse artigo foi construída a partir de relatórios internos e informações constantes no sistema de gestão da EMBRAPA. Valendo de regressões múltiplas e tendo como variáveis de desempenho, o número de tecnologias geradas, o número de artigos produzidos e o impacto das publicações originadas de cada projeto, o trabalho reveste-se de importância na medida em que aborda um tema latente para as Instituições Públicas de Pesquisa (IPP), qual sejam os fatores ligados ao desempenho de seus projetos e a distância entre intenção estratégica e resultado observado. 
Com efeito, para Sales-Filho (2000) o maior desafio para as IPPs tem sido conectar o estratégico ao operacional na implantação do modelo de planejamento e programação de pesquisa. Sob o ponto de vista teórico, os achados de nossa pesquisa quantitativa exploram o papel das competências acumuladas (Teece, 2010) e do esforço de articulação entre diferentes atores numa perspectiva de rede sobre o desempenho organizacional (Uzzi, 1997).

O trabalho estrutura-se da seguinte forma. Primeiramente, discorre-se sobre as relações entre os ferramentais da área de estratégia passíveis de serem apropriados no processo de inovação. Por questões de foco, exploram-se algumas lentes teóricas capazes de iluminar os fatores que podem afetar o desempenho dos projetos. Hipóteses são deduzidas a partir da discussão de temas relevantes da área de Estratégia em Organizações e com implicações diretas sobre atividades inovadoras, tais como: heterogeneidade de recursos, rendas relacionais e incentivos disponíveis à equipe de execução do projeto. Na sequência, são apresentados os procedimentos metodológicos utilizados nesse estudo. Por fim, apresentam-se os principais resultados e as conclusões do artigo.

\section{USANDO AS LENTES DA ESTRATÉGIA PARA COMPREENDER A DINÂMICA DE INOVAÇÃO}

A inovação é a mola mestra que altera as formas de organização do trabalho e possibilita a abertura de novos mercados. Resultante de novas combinações dos fatores de produção, a inovação está associada a novos processos organizacionais ou a novos produtos e serviços (Schumpeter, 1982). Recentemente, a literatura na área de administração tem dado uma atenção especial às relações entre estratégia e inovação (Tecce, 2010). A dinâmica dos mercados, as mudanças constantes nas cadeias produtivas e a necessidade de decisões empresariais em condições de incerteza têm convidado as organizações a pensar estratégia não como um padrão deliberado estático, mas sim como um processo emergente e dinâmico, capaz, inclusive, de lidar com a tendência ao desequilíbrio.

Em seu turno, as decisões estratégicas podem analisadas por meio de lentes distintas. Enquanto Porter (1980) concentrou seus estudos nas forças que moldam a estrutura dos setores e que determinam a competitividade, considerando mais enfaticamente fatores ambientais, e Peng (2009) preferiu creditar às interações institucionais grande importância estratégica, Barney (1991) trouxe para dentro da organização essa perspectiva, considerando capacidades - integração de um conjunto de recursos no âmbito da firma, como determinantes de vantagem competitiva sustentável. O autor destacou que os recursos podem ser convenientemente classificados em três categorias: recursos de capital físico,

Revista de Administração e Inovação, São Paulo, v. 12, n.4 p. 119-139, out./dez. 2015. 
recursos de capital humano, e recursos de capital organizacional. Recurso de capital físico inclui a tecnologia física usada em uma empresa, que a empresa instala e equipa, em localizações geográficas, e seu acesso às matérias-primas. Recurso de capital humano inclui o treinamento, a experiência, julgamento, inteligência, relacionamento, e a perspicácia individual dos dirigentes e trabalhadores em uma empresa. Recurso de capital organizacional inclui: empresa formal com estruturados relatórios, planos formais e informais, controle, e seu sistema coordenado, como bem como formais relações entre grupos dentro da empresa e entre a empresa e aqueles em seu ambiente (Barney, 1991). A combinação de tais recursos numa perspectiva dinâmica passa a ser imperativo para o desempenho de atividades inovativas (Teece \& Pisano, 1994).

Numa tentativa de aproximar as teorias da estratégia da área de inovação, Zawislak et al. (2012) apresentam a capacidade de inovação como uma meta-capacidade, que é resultado de outras quatro capacidades, que são ao mesmo tempo diferentes, mas complementares. São elas: i) capacidade de desenvolvimento tecnológico - habilidade que qualquer firma tem de interpretar, absorver e eventualmente transformar uma dada tecnologia; ii) capacidade operacional - habilidade de executar, de forma eficiente, a capacidade produtiva da empresa de acordo com as rotinas, conhecimentos e técnicas em um dado momento; iii) capacidade gerencial - habilidade de transformar o resultado do desenvolvimento tecnológico em operações coerentes; e iv) capacidade transacional - habilidade de reduzir os custos de transação em marketing, barganha e, logística.

Partindo, portanto, dessa tipologia, podemos inferir que as hipóteses desse trabalho estão dispostas a discutir heterogeneidade de recursos, como uma característica que pode dotar um projeto de determinado desempenho por diferenças na capacidade operacional e na capacidade de desenvolvimento tecnológico. De igual sorte, parcerias, redes e alianças, constituem-se em fatores que podem impactar no desempenho de projetos a partir de diferenças nos atributos transacionais. Por fim, as hipóteses aqui derivadas levam em conta os incentivos para a construção de capital reputacional, compreendendo-os como elementos que podem determinar desempenho idiossincrático em atividades inovativas. As hipóteses serão pensadas à luz de organizações inovativas que possuem como produtos de sua atividade: a geração de tecnologias, a publicação dos achados das pesquisas na forma de artigos científicos, bem como os impactos de tais publicações. 


\subsection{Desempenho em equipes heterogêneas}

Além da vantagem estratégica estabelecida com recursos heterogêneos, melhores desempenhos em equipes são observados empiricamente quando da ocorrência da heterogeneidade informacionalfuncional, conforme Van Knippenberg e Schippers (2007). Essa heterogeneidade carrega atributos menos visíveis, tais como as relacionadas com experiências funcionais.

Segundo Puente-Palacios et al. (2008), pesquisas baseadas na perspectiva informacionalfuncional encontraram resultados diferentes, mostrando que os grupos heterogêneos apresentam melhores desempenhos se comparados aos homogêneos. Van Knippenberg e Schippers (2007) destacaram que o fundamento dessa concepção está na ênfase dada à diversidade para a execução do trabalho. Para os autores quanto maior a diversidade de pontos de vista, maior a probabilidade de se encontrarem respostas efetivas em decorrência da amplitude de habilidades, conhecimentos, competências sustentadas pelos membros. Os autores constataram ainda que existem evidências empíricas demonstrando que os grupos heterogêneos precisam refletir sobre a informação mais relevante devido a uma grande quantidade de visões, e isso previne que o grupo opte por uma decisão menos importante apenas por conta de um aparente consenso entre os membros do grupo. E como resultado importante para o presente trabalho, os autores inferem que a exposição a perspectivas divergentes auxilia no surgimento de ideias mais criativas e inovadoras.

O pensamento de Kurtzberg (2005) converge para o mesmo achado, de que equipes diversas têm uma maior capacidade de inovação e solução de problemas. $\mathrm{O}$ estudo realizado por esse autor revela que o afeto positivo dos indivíduos em relação à equipe pode potencializar a percepção de sucesso e de criatividade, incentivando os membros do grupo a superarem as expectativas de desempenho. Assim, na presença de resultados favoráveis de desempenho, equipes caracterizadas pela diversidade possuem a elevada probabilidade de ocorrência de afetos positivos, os quais, por sua vez, promovem visões positivas sobre os resultados e, posteriormente, resultados de desempenho efetivamente positivos. Ratificando e consolidando a dualidade de resultados obtidos em estudos sobre a composição das equipes de trabalho, Horwitz (2005) apresenta duas vertentes teóricas antagônicas que acompanham os resultados apresentados pelos autores citados anteriormente. É apresentado o paradigma da atração similaridade, que considera atributos observáveis e segundo o qual equipes heterogêneas são negativamente relacionadas com desempenho e integração social. Nessa perspectiva, a homogeneidade se daria em função da atração imediata proporcionada pelas similaridades compartilhadas pelas pessoas. A outra proposta teórica é a da Diversidade do Recurso Cognitivo. Segundo ela as dimensões de diversidade relevantes não são as imediatamente observáveis e envolvem

Revista de Administração e Inovação, São Paulo, v. 12, n.4 p. 119-139, out./dez. 2015. 
multiplicidade de formação profissional e as possibilidades singulares que cada indivíduo traz para a equipe, conferindo, a essa unidade de desempenho, maior capacidade de inovação, criatividade, resoluções de problemas e favorecendo a tomada de decisão por proporcionar uma base de informações mais diversificada.

Assim, uma vez que a heterogeneidade parece favorecer a inovação, e, considerando a geração de tecnologia e de conhecimento, caminhos iniciais determinantes à contribuição inovativa no âmbito de empresas inovadoras, tem-se as seguintes hipóteses:

\section{$H_{1 a}$ - Equipes heterogêneas de pesquisa são associadas a uma maior geração de tecnologias;}

H1b - Equipes heterogêneas de pesquisa são associadas a uma maior publicação de artigos científicos;

H1c - Equipes heterogêneas de pesquisa são associadas a um maior impacto da geração de conhecimento na comunidade científica.

\subsection{Parcerias, Redes e Alianças e Rendas Relacionais}

A literatura da área de estratégia sustenta que a produção em rede com mecanismos de transferência de conhecimentos entre usuários, fornecedores e fabricantes possui maiores chances de promover uma inovação aberta. Estes estudos sugerem que a aliança de uma empresa com parceiros é, em muitos casos, a mais importante fonte de novas ideias que resultem na melhoria de desempenho de tecnologias e inovações (Uzzi, 1997; Cassiman \& Veugelers, 2006).

A formação de parcerias, por seu turno, pode favorecer melhores desempenhos ao tempo que permitem a complementaridade de recursos, redução da assimetria de informações e o compartilhamento de conhecimento. Dyer e Singh (1998) apontam que os ganhos de produtividade na cadeia de valor são possíveis quando os parceiros estão dispostos a fazer uma relação de investimentos específicos e combiná-los de forma única. Assim, ligações entre entes idiossincráticos podem ser uma fonte de renda relacional e de vantagem competitiva. Rendas relacionais, para os autores, consistem em rendas decorrentes de empresas que combinam recursos de forma única. Os autores ainda a definem como um lucro supernormal gerado em conjunto numa relação de troca que não pode ser gerado por qualquer empresa isoladamente, mas sim através de contribuições (idiossincráticas) de parceiros na aliança.

Revista de Administração e Inovação, São Paulo, v. 12, n.4 p. 119-139, out./dez. 2015. 
As rendas relacionais são possíveis quando parceiros de alianças combinam, trocam ou investem em bens, conhecimentos, recursos e mecanismos de governança eficazes que reduzem os custos de transação. Redes de relacionamento que partilham conhecimento podem influenciar o desempenho da atividade (Gulati et al 2000). De igual sorte, a capacidade de absorção específica dos parceiros influencia o potencial para geração de rendas relacionais e de inovações estratégicas (Gebauer et al 2012). Para tal, o alinhamento de incentivos para que os parceiros sejam transparentes na transferência de conhecimentos é fundamental. Estes incentivos podem ser financeiros ou normas informais de reciprocidade (Dyer \& Singh, 1998).

Existem evidências teóricas, portanto, das rendas relacionais que podem ser obtidas através da realização de parcerias com indivíduos e organizações situadas além dos limites da organização de referência analisada. Para a inovação, redes e alianças funcionam como estratégia que pode conduzir a desempenhos importantes, no caminho da inovação. Particularmente, no setor de pesquisa agropecuária, essas relações podem ser observadas quando grupos de pesquisadores de uma unidade se relacionam com outros indivíduos pertencentes a outras redes de pesquisa. Assim:

\section{$H_{2 a}$ - A presença de pesquisadores externos à Unidade favorece a geração de tecnologias; $H_{2 b}$ - A presença de pesquisadores externos à Unidade favorece a publicação de artigos científicos;}

\section{$\mathbf{H}_{2 c}$ - A presença de pesquisadores externos à Unidade favorece o maior impacto da geração de conhecimento na comunidade científica.}

\subsection{O papel da Reputação e dos Incentivos}

Ao defender que os atores estão imersos em redes de relações sociais, Granovetter (2007) asseverou que os atores não se comportam nem tomam decisões como átomos fora de um contexto social, e nem adotam de forma servil um roteiro escrito para eles pela intersecção específica das categorias sociais que eles porventura ocupem. Nesse contexto, relações pessoais concretas, redes de relacionamento são importantes para o aumento da confiança, para o estímulo à cooperação e para o fortalecimento de questões. Uzzi (1997) estabeleceu também o desempenho econômico como sendo derivado da imersão social. No seu estudo etnográfico, ele constatou que os laços são desenvolvidos principalmente a partir de redes de referência e de relações pessoais anteriores. Em outras palavras, o autor esclarece que laços imersos muitas vezes são estabelecidos nas relações porque as pessoas se conhecem de outros círculos sociais como colaboradores, colegas, amigos, parentes. As redes de 
referências e os vínculos pessoais facilitam o surgimento de laços mediante a aplicação de oportunidades e expectativas de relações preexistentes incorporados a novos relacionamentos e situações.

No âmbito de projetos de pesquisa e desenvolvimento, os incentivos conferidos aos pesquisadores parecem jogar um papel importante no desempenho das atividades (Hoskisson, Hitt $\&$ Hill, 1993). Indivíduos podem, nessa seara, serem incentivados para realizar esforços adicionais que resultarão em desempenho distintivo e consequentemente em reputação adicional capaz de atrair novos parceiros. Há outro efeito colateral nesse mecanismo, os agentes tenderiam a fugir do custo dos danos infligidos à reputação pessoal em caso de mau desempenho. Mecanismos de premiação podem ser invocados para estimular o comportamento desejado (Roberts, 2010).

Assim, no contexto do presente trabalho em que pesquisadores podem ser beneficiados por programas que conferem visibilidade, reputação e prestígio, como por exemplo, o status de pesquisadores de agências de fomento, e com isso alavancarem suas produções e seus impactos relativos, têm-se as seguintes proposições:

\section{H3a - A presença de pesquisadores com maior prestígio acadêmico está associada a uma maior geração de tecnologias;}

$H_{3 b}$ - A presença de pesquisadores com maior prestígio acadêmico está associada a uma maior publicação de artigos científicos;

H3c - A presença de pesquisadores com maior prestígio acadêmico está associada a um maior impacto da geração de conhecimento na comunidade científica.

\section{DADOS E MÉTODO}

O presente estudo tomou como unidade de análise um centro de pesquisa da Embrapa, empresa pública vinculada ao Ministério da Agricultura, Pecuária e Abastecimento e criada em 26 de abril de 1973, cuja missão é viabilizar soluções de pesquisa, desenvolvimento e inovação para a sustentabilidade da agricultura, em benefício da sociedade brasileira.

A base de dados foi construída com 40 projetos liderados pela Embrapa Mandioca e Fruticultura, e abrangeu a totalidade dos projetos concluídos na unidade analisada entre os anos de 2005 e 2012, registrados na base de dados da Embrapa.

Revista de Administração e Inovação, São Paulo, v. 12, n.4 p. 119-139, out./dez. 2015. 
Os dados coletados foram analisados a partir de análise multivariada, onde regressões foram utilizadas com o auxílio do software Stata 11.

No modelo de análise foram utilizadas como variáveis dependentes o "número de tecnologias geradas", o "número de artigo publicados em periódicos" e o "impacto no Journal of Citation Reports (JCR) dos artigos publicados em periódicos". Como variáveis independentes, foram utilizadas: "Desvio padrão do tempo de empresa dos pesquisadores" e "Número de especialidades acadêmicas na equipe do projeto" como indicadores de Heterogeneidade das Equipes; "Percentual de pesquisadores externos ao Centro Nacional de Pesquisa em Mandioca e Fruticultura (CNPMF)" como indicador de Parcerias; e "Percentual de bolsistas de produtividades por projeto" como indicador de Prestígio Acadêmico associado à Reputação.

Como variáveis de controle, consideramos "Número de pesquisadores envolvidos no projeto", "Percentual de pesquisadores com título de doutor ou PHD", "Percentual de Recursos Extratesouro", "Valor dos recursos financeiros do contrato" e "Macroprogramas 4 e 5".

A tabela 1 apresenta os resultados das estatísticas descritivas das variáveis estudadas no presente trabalho.

Tabela 1 - Resultados da estatística descritiva das variáveis analisadas

\begin{tabular}{ccccccc}
\hline Descrição & $\begin{array}{c}\text { Tipo de } \\
\text { Variável }\end{array}$ & Obs. & Média & $\begin{array}{c}\text { Desvio } \\
\text { Padrão }\end{array}$ & Mínimo & Máximo \\
\hline $\begin{array}{c}\text { Número de tecnologias } \\
\text { geradas por projeto }\end{array}$ & $\begin{array}{c}\text { Dependente } \\
\left(\mathrm{Y}_{1}\right)\end{array}$ & 40 & 0,70 & 1,45 & 0,00 & 8,00 \\
$\begin{array}{c}\text { Número de artigos gerados } \\
\text { por projeto }\end{array}$ & $\begin{array}{c}\text { Dependente } \\
\left(\mathrm{Y}_{2}\right)\end{array}$ & 35 & 3,14 & 6,43 & 0,00 & 28,00 \\
$\begin{array}{c}\text { Soma dos índices de impacto } \\
\text { "JCR" dos artigos publicados } \\
\text { em periódicos }\end{array}$ & $\begin{array}{c}\text { Dependente } \\
\left(\mathrm{Y}_{3}\right)\end{array}$ & 35 & 2,39 & 5,10 & 0,00 & 21,52 \\
$\begin{array}{c}\text { Desvio Padrão do tempo de } \\
\text { empresa dos pesquisadores }\end{array}$ & $\begin{array}{c}\text { Independente } \\
(\mathrm{H} 1)\end{array}$ & 40 & 8,59 & 4,71 & 0,00 & 18,54 \\
$\begin{array}{c}\text { Número de especialidades } \\
\text { acadêmicas }\end{array}$ & $\begin{array}{c}\text { Independente } \\
(\mathrm{H} 1)\end{array}$ & 40 & 3,53 & 3,79 & 1,00 & 24,00 \\
$\begin{array}{c}\text { Percentual de pesquisadores } \\
\text { externos ao CNPMF }\end{array}$ & $\begin{array}{c}\text { Independente } \\
(\mathrm{H} 2)\end{array}$ & 40 & 0,18 & 0,27 & 0,00 & 0,95 \\
$\begin{array}{c}\text { Percentual de bolsistas de } \\
\text { produtividades por projeto }\end{array}$ & $\begin{array}{c}\text { Independente } \\
(\mathrm{H} 3)\end{array}$ & 40 & 0,19 & 0,26 & 0,00 & 1,00 \\
$\begin{array}{c}\text { Número de pesquisadores } \\
\text { envolvidos no projeto }\end{array}$ & $\begin{array}{c}\text { Controle } \\
40\end{array}$ & 8,75 & 14,38 & 1,00 & 86,00
\end{tabular}




\begin{tabular}{ccccccc}
\hline Descrição & $\begin{array}{c}\text { Tipo de } \\
\text { Variável }\end{array}$ & Obs. & Média & $\begin{array}{c}\text { Desvio } \\
\text { Padrão }\end{array}$ & Mínimo & Máximo \\
\hline $\begin{array}{c}\text { Percentual de pesquisadores } \\
\text { com título de doutor ou PHD }\end{array}$ & Controle & 40 & 0,80 & 0,21 & 0,29 & 1,00 \\
$\begin{array}{c}\text { Percentual de Recursos } \\
\text { extratesouro }\end{array}$ & Controle & 40 & 0,52 & 0,46 & 0,00 & 1,00 \\
$\begin{array}{c}\text { Valor dos recursos } \\
\text { financeiros do contrato } \\
\text { Macroprogramas 4 e 5 }\end{array}$ & Controle & 40 & $184.639,50$ & $272.317,30$ & $21.790,00$ & $\begin{array}{c}1.438 .926, \\
00\end{array}$ \\
\hline
\end{tabular}

Fonte: Resultados da pesquisa.

A análise das estatísticas descritivas das variáveis dependentes sugere algumas reflexões. No quesito "número de tecnologias geradas", os 40 projetos observados geraram em média 0,70 tecnologias. Existem projetos de pesquisa científica que, pela própria natureza de sua concepção, do seu tema, não possuem muitas perspectivas de gerar tecnologias.

Deve ser observado que a variável "número de artigos gerados por projeto" apresentou 5 missing values, totalizando 35 observações. Tal fato se deve à ausência de informações relacionadas à quantidade de artigos gerados em cinco projetos de pesquisa. Para evitar a perda de graus de liberdade nas análises quantitativas realizadas, buscou-se complementar tais informações por meio de interações junto aos líderes de projeto, porém diante da impossibilidade de se obter um registro confiável, optouse por executar as análises somente com as informações presentes nas bases oficiais da organização estudada. Observa-se que a quantidade de artigos publicados, em média, é maior do que a quantidade de tecnologias geradas. Possivelmente, isso se deve ao fato de a Embrapa promover uma política de incentivos (promoção e premiação) atrelada a indicadores como publicações de artigos periódicos indexados, ou não.

Em relação à "Soma dos índices de impacto "JCR" dos artigos publicados em periódicos", percebe-se uma variação importante. Enquanto um projeto apresentou uma soma de 21,52, outro projeto não apresentou impacto algum. Com um desvio padrão de 5,10, a média da soma dos impactos dos artigos publicados em periódicos ficou em 2,39. Uma observação importante, é que o projeto que teve maior soma de JCR, com 21,52 pontos, foi um projeto em que todos os artigos foram publicados em inglês, em sua grande maioria em revistas internacionais. As regressões revelam quais são fatores que estão significantemente relacionados a tais padrões de desempenho. 


\subsection{Procedimento de Análise}

A equação a seguir demonstra o modelo de análise quantitativa customizada para a pesquisa:

$$
\mathrm{Y}_{\mathrm{i}, \mathrm{d}}=\mathrm{f}\left(\Upsilon_{\mathrm{i},,}, \beta_{\mathrm{i},}, \Omega_{\mathrm{i}}, \alpha_{\mathrm{i}}\right)(1)
$$

Na equação (1), o indicador de desempenho " $d$ " de um determinado projeto "i” pode ser exprimido em função das diversas variáveis independentes escolhidas. Os vetores $\Upsilon_{\mathrm{i},}, \beta_{\mathrm{i},}, \Omega_{\mathrm{i}}, \alpha_{\mathrm{i}}$ refletem respectivamente aspectos relacionados a heterogeneidade de recursos, rendas relacionais, incentivos/reputação e varáveis de controle, as quais em conjunto podem afetar o desempenho observado.

As características dos dados determinaram cuidados na determinação do modelo. Seguindo a sugestão de McCullagh e Nelder (1989), utilizou-se o modelo linear generalizado de Poisson na análise das variáveis dependentes "Geração de Tecnologias" e "Geração de Conhecimento Científico". Tal escolha se justifica pelo fato dessas duas variáveis dependentes serem oriundas de dados de contagem (count data), representarem eventos raros e com grande incidência de zeros. Nessas condições, um modelo linear clássico tende a não proporcionar o melhor ajuste. Conforme apontado por Anderson et al (2002) e Cabral (2006), o reduzido número de observações da amostra não compromete a robustez dos resultados em modelos de Poisson caso o tamanho da amostra seja igual ou superior a 30 observações e caso os dados sejam aderentes a distribuição de Poisson. Tais características são observadas no presente trabalho. Por fim, o modelo de Poisson tem sido largamente utilizado em pesquisas na área de Administração no Brasil em seus mais diversos campos, incluindo as áreas de estratégia e políticas públicas (Cabral, Lazzarini e Azevedo, 2010; Cabral e Lazzarini, 2010), comportamento do consumidor (Spers, Zylberstajn e Lazzarini, 2003) e finanças (Kirsch, Lima e Terra, 2012).

No entanto, para as análises com a variável dependente "Impacto do Conhecimento Científico Gerado", foi utilizado o modelo Mínimos Quadrados Ordinários (MQO). Os modelos de Mínimos Quadrados Ordinários são utilizados para analisar tendências de variáveis dependentes quantitativas com nível de mensuração intervalar (os dados não possuem um ponto inicial zero natural) ou de razão (é similar ao intervalar, mas há um ponto inicial zero natural). 


\section{ANÁLISE E DISCUSSÃO DOS RESULTADOS}

A partir das análises geradas conforme metodologia descrita anteriormente, resultados importantes foram encontrados. O exame da tabela 2 permite observar os fatores influenciadores do desempenho nas três variáveis analisadas. Discutimos cada uma delas a seguir.

Tabela 2

Resultados para os indicadores Número de Tecnologi as Geradas, Número de Artigos Gerados e Impacto dos Artigos (JCR) : Estimativas Obtidas por meio de Regressões de Poiss on e MQO*

\begin{tabular}{|c|c|c|c|c|c|c|}
\hline & \multicolumn{3}{|c|}{ Número de tecnologias } & \multicolumn{2}{|c|}{$\begin{array}{l}\text { Número de artigos } \\
\text { (JCR) }\end{array}$} & \multirow{2}{*}{$\begin{array}{l}\text { Impacto dos Artigos } \\
\text { Modelo } 6\end{array}$} \\
\hline & Mod elo 1 & Modelo 2 & Modelo 3 & Modelo 4 & Modelo 5 & \\
\hline $\begin{array}{l}\text { Desvio Padrão } \\
\text { do tempo de } \\
\text { empresa dos }\end{array}$ & & $0,196^{* * *}$ & & $-0,044$ & & $-0,130$ \\
\hline pesquisadores & & $(0,068)$ & & $(0,042)$ & & $(0,197)$ \\
\hline $\begin{array}{l}\text { Número de } \\
\text { especialidades }\end{array}$ & & $0,251 * * *$ & & $-0,264 * * *$ & & $-0,583$ \\
\hline acadêmicas & & $(0,095)$ & & $(0,049)$ & & $(0,642)$ \\
\hline $\begin{array}{l}\text { Percentual de } \\
\text { pesquisadores } \\
\text { externos ao }\end{array}$ & & $2,761 * *$ & & 1,149 & & 0,651 \\
\hline CNPMF & & $(1,171)$ & & $(1,776)$ & & $(5,140)$ \\
\hline $\begin{array}{l}\text { Percentual de } \\
\text { bolsistas de } \\
\text { produtividades }\end{array}$ & & $-4,208 *$ & & 1,588 & & 0,875 \\
\hline por projeto & & $(2,235)$ & & $(1,358)$ & & $(4,215)$ \\
\hline $\begin{array}{l}\text { Número de } \\
\text { pesquisadores } \\
\text { envolvidos no }\end{array}$ & $0,028 * * *$ & $-0,066^{* *}$ & $0,022 * * *$ & $0,087^{* * *}$ & 0,083 & 0,227 \\
\hline projeto & $(0,005)$ & $(0,031)$ & $(0,008)$ & $(0,019)$ & $(0,060)$ & $(0,185)$ \\
\hline $\begin{array}{l}\text { Percentual de } \\
\text { pesquisadores } \\
\text { com titulo de }\end{array}$ & $-1,383$ & $-0,741$ & $-0,490$ & $-0,117$ & 0,831 & $-0,112$ \\
\hline doutor ov PHD & $(1,154)$ & $(1,173)$ & $(1,328)$ & $(1,212)$ & $(4,041)$ & $(4,931)$ \\
\hline $\begin{array}{c}\text { Percentual de } \\
\text { Recursos }\end{array}$ & $-1,108$ & $-2,452^{* * *}$ & 0,190 & 1,516 & 1,545 & 2,103 \\
\hline extratesouro & $(0,903)$ & $(0,628)$ & $(0,601)$ & $(1,027)$ & $(2,004)$ & $(2,203)$ \\
\hline $\begin{array}{l}\text { Log do valor } \\
\text { dos recurs os }\end{array}$ & 0,014 & $-0,213$ & $0,590 * * *$ & $0,764 * *$ & $2,089 * *$ & $2,175^{*}$ \\
\hline $\begin{array}{l}\text { financeiros do } \\
\text { contrato }\end{array}$ & $(0,228)$ & $(0,237)$ & $(0,207)$ & $(0,346)$ & $(0,941)$ & $-1,151$ \\
\hline $\begin{array}{c}\text { Macroprograma } \\
\text { s4 e } 5\end{array}$ & $\begin{array}{l}-1,766 * \\
(0,949) \\
\end{array}$ & $\begin{array}{l}-2,450 * \\
(1,353) \\
\end{array}$ & $\begin{array}{c}- \\
17,510 * * * \\
(0,532)\end{array}$ & $\begin{array}{c}- \\
17,512 * * * \\
(0,689)\end{array}$ & $\begin{array}{l}-3,366 \\
(2,055) \\
\end{array}$ & $\begin{array}{l}-3,208 \\
(2,275) \\
\end{array}$ \\
\hline Intercepto & $\begin{array}{c}0,617 \\
(3,157) \\
\end{array}$ & $\begin{array}{c}1,029 \\
(2,915)\end{array}$ & $\begin{array}{c}-5,793^{* *} \\
(2,917) \\
\end{array}$ & $\begin{array}{l}-8,743^{*} \\
(4,480)\end{array}$ & $\begin{array}{l}-23,250^{*} \\
(11,955) \\
\end{array}$ & $\begin{array}{l}-22,095 \\
(13,827)\end{array}$ \\
\hline Obs, & 40 & 40 & 40 & 40 & 40 & 40 \\
\hline
\end{tabular}

Nota. Niveis de significância utilizados: *=10\%; **=5\%; ***=1\%, Erros-padrão em parênteses. 


\subsection{Impacto sobre o número de tecnologias geradas}

A variável Desvio Padrão do tempo de empresa dos pesquisadores, associada à hipótese H1a, mostrou apresentar uma relação positiva e altamente significante com o número de tecnologias geradas. Numa equipe de projeto, quanto maior o desvio padrão do tempo de empresa dos pesquisadores existe uma probabilidade grande de se gerar mais tecnologias. $\mathrm{O}$ desvio padrão indica a dispersão do tempo de empresa dos empregados e quanto maior o desvio padrão na equipe do projeto, mais os projetos terão pesquisadores novos trabalhando juntos de pesquisadores seniores, sendo uma proxy de heterogeneidade de recursos e de equipes, característica que pode levar a vantagem competitiva (Barney, 1991). A diferença de tempo de empresa oferece pistas para se inferir relações distintas entre esses indivíduos. Um desvio padrão de 18,54 anos, por exemplo, ponto máximo da amostra, permite pensar que existem indivíduos com formações completamente diferentes. A união, portanto, do novo - das inovações científicas recentes, do comportamento mais curioso, da experimentação de novos processos, novas tecnologias, novas teorias, da vontade de produzir os primeiros resultados, trazido pelos pesquisadores novos, com a experiência, o know how, o aprendizado acumulado, as capacidades transacionais desenvolvidas, as redes e alianças mais sólidas, desenvolvidas pelos pesquisadores seniores, tem forte correlação com o desempenho, no que tange à geração de um número maior de tecnologias. Dessa forma, esse primeiro indicador da variável heterogeneidade, confirma a hipótese H1a.

Alternativamente, utilizamos o número de especialidades acadêmicas para estimar a heterogeneidade. Com uma positiva correlação e bastante significativa estatisticamente, confirmou-se a H1a também por esse indicador: equipes heterogêneas de pesquisa são associadas a uma maior geração de tecnologias agropecuárias. Assim quanto mais especialistas de diversas áreas atuam na solução tecnológica maior a probabilidade dessa tecnologia ser gerada. Boa parte dos problemas que demandam uma tecnologia é de alta complexidade e para dar cabo da complexidade, quanto mais capacidades nas mais diversas possibilidades de solução, maiores são as chances de se chegar a um resultado, de modo que a multiplicidade de formação profissional da equipe parece conferir maior capacidade de inovação.

Para testar a segunda hipótese, H2a, utilizamos como proxy de aspectos relacionais o percentual de pesquisadores externos ao organismo em análise, partindo do princípio que a complementação de conhecimentos tácitos e a troca das experiências locais são consequência das relações de parcerias estabelecidas entre pesquisadores de outras Unidades que estão claramente influenciando positivamente e significativamente na variável estudada. Fica evidente, nessa análise, 
que o desempenho dos projetos, no que diz respeito a geração de tecnologia, está sendo influenciado significativamente pelas rendas relacionais provenientes das parcerias construídas $(\mathrm{p}<0.05)$, confirmando assim a hipótese de pesquisa $\mathrm{H} 2 \mathrm{a}$.

Finalmente, a variável Percentual de bolsistas de produtividades por projeto, busca capturar aspectos reputacionais como forma de testar a hipótese H3a. Observa-se na tabela 2 que há significância de uma forte correlação negativa entre o percentual de bolsistas de produtividade e o número de tecnologias geradas. Desse resultado, pode-se deduzir, que como o alto status conferido pela bolsa de produtividade não implica em maior geração de tecnologias, de modo que há um trade off entre produção tecnológica e prestígio acadêmico dos pesquisadores envolvidos. Isso se deva, talvez, pelo fato da progressão na carreira de pesquisador estar associada à excelência continuada na produção científica e na formação de recursos humanos. Os pesquisadores, nesse caso, estariam apenas respondendo aos incentivos que foram submetidos (Roberts, 2010). A existência dessa incompatibilidade não permite confirmar a hipótese H3a.

\subsection{Impacto sobre o número de artigos científicos publicados}

Quando se avalia o impacto de nossas variáveis preditoras sobre o número de artigos científicos publicados, observa-se comportamento relativamente distinto. O exame da variável "Desvio Padrão do tempo de empresa dos pesquisadores”, associada à hipótese $\mathrm{H} 1 \mathrm{~b}$, demonstra que não há significância estatística que possa assegurar que a heterogeneidade de recursos leva a produção de artigos. Aparentemente, a mescla entre pesquisadores novos e seniores não alavanca a produção científica, não permitindo a confirmação da hipótese H1b.

No entanto, quando avaliamos a influência do "Número de especialidades acadêmicas", que também captura a heterogeneidade de recursos e está associada à H1b, apresentou uma forte correlação negativa $(\mathrm{p}<0.01)$. Contrariando as expectativas iniciais, uma maior homogeneidade de especialidades acadêmicas na equipe do projeto parece contribuir para uma maior geração de artigos científicos. Tal fato pode ser explicado, talvez, pela dificuldade que pesquisadores de diversas áreas do conhecimento possuem para atuar conjuntamente e construir um texto científico multidisciplinar, ao menos em nosso contexto empírico fortemente marcado por revistas de impacto de caráter disciplinar (i.e Plant Disease, Archives of Virology, BMC Genomics, Genetics and Molecular Biology, Summa Phytopathologica, Revista Brasileira de Solos, Fitopatologia Brasileira, dentre outras). A especificidade presente parece impedir a produção científica conjunta em equipes heterogêneas. Dessa 
forma, podemos afirmar que não foi possível confirmar a H1b para o indicador de desempenho relacionado à produção de artigos.

A inspeção das demais variáveis mostra que os aspectos relacionais não apresentam relação com os artigos publicados, assim como o prestígio de pesquisadores, inviabilizando a confirmação das hipóteses $\mathrm{H} 2 \mathrm{~b}$ e H3b. Entretanto, ao observar o efeito das variáveis de controles sobre a produção científica, observamos uma forte relação da quantidade de pesquisadores envolvidos no projeto e do montante de recursos destinados com o número de artigos publicados (ambos a p<0.01). Porém, projetos debruçados sobre os Macroprogramas 4 e 5 influenciam negativamente a relação de artigos publicados. Os Macroprogramas 4 e 5 são destinados a Projetos de Comunicação e Transferência de Teconologia e Projetos de Desenvolvimento Institucional respectivamente, que pela sua própria natureza, não têm caracterísitcas de gerar tecnologias e conhecimento científico. O controle desses aspectos por meio dessa variável é essencial para que se possa diminuir a influência de fatores não observáveis nas relações de causalidade analisadas.

\subsection{Efeito sobre o impacto dos artigos (Journal Citation Reports)}

A observação da Tabela 2 permite verificar os fatores que influenciam o impacto das produções científicas oriundas dos projetos de pesquisa. Tendo como proxy de desempenho a variável dependente "Impacto da Geração de Conhecimento na Comunidade Científica", verifica-se que a heterogeneidade da equipe não afeta o impacto da produção científica realizada pelo grupo, uma vez que as variáveis Desvio Padrão do tempo de empresa dos pesquisadores 3 e Número de especialidades acadêmicas não são estatisticamente significativas. Desse modo, não foi possível confirmar a H1c.

O exame da variável Percentual de pesquisadores de outras unidades/instituições, associada à hipótese $H 2 c$, revela igualmente que a relação entre a formação de redes de colaboração e o impacto das publicações parece não ocorrer no contexto estudado. Surpreendentemente, a presença de pesquisadores de prestígio no projeto não tem contribuído para aumentar o impacto da produção cientifica qualificada com fator JCR. Ou seja, em que pese a existência de um trade-off entre produção científica e produção tecnológica, conforme já discutido, observa-se que os esforços da produção científica de pesquisadores mais renomados não têm sido materializados em publicações renomadas. Infelizmente, não dispomos de medidas para avaliar se tal resultado é uma característica da organização examinada ou se é algo que perpassa as instituições de pesquisa como um todo. De toda a sorte, ao menos no caso em tela, não foi possível confirmar as $\mathrm{H} 2 \mathrm{c}$ e $\mathrm{H} 3 \mathrm{c}$.

Revista de Administração e Inovação, São Paulo, v. 12, n.4 p. 119-139, out./dez. 2015. 
O quadro abaixo sumariza os principais achados desse trabalho.

Quadro 1 - Quadro resumo do resultado do teste das hipóteses de trabalho

\begin{tabular}{|c|c|}
\hline HIPÓTESES & RESULTADOS \\
\hline $\begin{array}{cc}\text { Heterogeneidade } & \text { Número de tecnologia } \\
\text { Número de artigos } \\
\text { Impacto dos artigos }\end{array}$ & $\begin{array}{l}\text { Suportada para número de tecnologias } \\
\text { geradas } \\
\text { Não suportada para número de artigos } \\
\text { científicos publicados } \\
\text { Não suportada para maior impacto dos } \\
\text { artigos }\end{array}$ \\
\hline $\begin{array}{l}\text { Número de tecnologias } \\
\text { Número de artigos } \\
\text { Impacto dos artigos } \\
\uparrow\end{array}$ & $\begin{array}{l}\text { Suportada para número de tecnologias } \\
\text { geradas } \\
\text { Não suportada para número de artigos } \\
\text { científicos publicados } \\
\text { Não suportada para maior impacto dos } \\
\text { artigos }\end{array}$ \\
\hline $\begin{array}{l}\text { Número de tecnologias } \\
\text { Número de artigos } \\
\text { Impacto dos artigos } \\
\uparrow\end{array}$ & $\begin{array}{l}\text { Não suportada para número de tecnologias } \\
\text { geradas } \\
\text { Não suportada para número de artigos } \\
\text { científicos publicados } \\
\text { Não suportada para maior impacto dos } \\
\text { artigos }\end{array}$ \\
\hline
\end{tabular}

Fonte: Elaborado pelos autores

\section{CONSIDERAÇÕES FINAIS}

O presente trabalho procurou abordar os fatores que podem influenciar o desempenho em projetos de inovação tecnológica. Valendo-se de uma instituição pública de pesquisa, as análises efetuadas com as lentes das teorias da área de estratégia apontam que equipes heterogêneas e a formação de parcerias, redes e alianças influenciam positivamente no número de tecnologias geradas pelos projetos de pesquisa. De igual sorte, observou-se que quanto maior o número de "bolsistas de produtividade CNPq na equipe do projeto" menor é o número de tecnologias geradas, sinalizando a ocorrência de um trade-off entre produções científicas e produção de cunho tecnológico. A pesquisa revela que quanto mais homogênea é a equipe e quanto maior o número de pesquisadores envolvidos no projeto, maior é o número de artigos científicos publicados. Surpreendentemente, identificou-se que 
as redes e alianças e a maior reputação dos pesquisadores da equipe do projeto não têm influenciado no número de artigos publicados. Adicionalmente às hipóteses de pesquisa aqui formuladas, observouse que o número de tecnologias geradas é influenciado negativamente pela presença de maior percentual de recursos extratesouro no montante de financiamento do projeto. Tal resultado pode servir de guia para as fundações de fomento, no que diz respeito aos possíveis efeitos adversos decorrentes de editais, na medida em que os achados sugerem impactos negativos na geração de tecnologias e inovação em projetos financiados com recursos externos. Isso pode se ocorrer pelo fato dos editais das agências possuírem prazos que giram em torno de 3 anos. Sob tais restrições temporais, talvez não seja possível aos pesquisadores obter contribuições voltadas a geração de tecnologias agropecuárias, que por sua natureza , por vezes, demandam longos períodos de experimentação. Destarte, os resultados sugerem que produzir artigos parece ser mais atrativo aos pesquisadores que obtém financiamento por meio de recursos extratesouro em relação a obtenção de produtos tecnológicos.

Ao contemplar o desempenho advindo de recursos heterogêneos com resultados positivos provenientes de rendas relacionais, o trabalho lança luzes sobre a influência de fatores abordados pelas teorias da área de estratégia sobre projetos de inovação tecnológica em áreas potencialmente negligenciadas pelo setor privado e que, por isso, apresentam forte influência governamental. Por meio de uma base de dados única debruçada sobre o desempenho de projetos de inovação e pesquisa agropecuária, o estudo ressalta o papel da interação intrainstitucional e de redes de colaboração em projetos de pesquisa.

Merece consideração abordar o que foi admitido como "desempenho" nesse trabalho. É claro que, à luz da estratégia, geração de tecnologia e geração de conhecimento científico podem ser considerados indicadores operacionais, finalísticos, que juntamente com as regressões apresentadas aqui já permitem subsidiar decisões gerenciais de alocação de recursos e apontar para algumas capacidades essenciais, por exemplo. Contudo, o resultado dessa pesquisa se propôs a um estudo micro organizacional, tendo como unidade de análise o projeto de pesquisa. Entende-se, portanto, que próximos estudos podem ser realizados levando em conta uma perspectiva mais ampla, como por exemplo, valor gerado a partir dos produtos obtidos. De uma forma mais clara, qualificar essas tecnologias, quanto ao seu serviço social, seu impacto econômico e suas implicações ambientais pode enriquecer a análise, sofisticando os indicadores, e, dessa forma, servir definitivamente como um mais robusto indicador estratégico.

O trabalho teve como limitação o número de observações. Outra limitação ficou a cargo da falta de absoluta certeza da exatidão das informações encontradas nos relatórios, alimentados pelos pesquisadores quando da submissão do projeto. Pode-se também considerar limitação, possível erro 
humano na alimentação da base de dados, apesar de toda atenção que foi dedicada a essa atividade durante o processo de coleta de dados. Sugere-se continuar com a formação de um painel, de forma que análises possam ser feitas periodicamente, visando observar o comportamento dinâmico das competências. Tal sugestão vai ao encontro da teoria das competências dinâmicas que considera o recurso não estático e sim mutante. Para a criação desse painel, sugere-se a revisão dos relatórios finais de projeto, de modo que eles contenham campos que permitam a formação de uma base de dados que contemple indicadores capazes de se fazer observar e documentar as mais importantes capacidades dos projetos de pesquisa. Em que pese tais limitações, os achados e conclusões aqui descritos apresentam implicações práticas relevantes que podem permitir ações gerenciais voltadas ao incremento do desempenho da atividade inovativa.

\section{REFERÊNCIAS}

Anderson, D. R., Sweeney, D. J., \& Williams, T. A. (2001). Statistiques pour l'économie et la gestion: De Boeck.

Barney, J. (1991). Firm resources and sustained competitive advantage. Journal of management, 17(1), 99-120.

Besanko, D., Dranove, D., Shanley, M., \& Schaefer, S. (2007). A economia da estratégia. Bookman.

Bryson, J. M., Ackermann, F., \& Eden, C. (2007). Putting the resource-based view of strategy and distinctive competencies to work in public organizations. Public Administration Review, 67(4), 702717.

Cabral, S. (2006). Além das grades: uma análise comparada das modalidades de gestão do sistema prisional. Doutorado, Universidade Federal da Bahia, Salvador

Cabral, S., \& Lazzarini, S. G. (2010). Impactos da participação privada no sistema prisional: evidências a partir da terceirização de prisões no Paraná. Revista de Administração Contemporânea, 14(3), 395-413.

Cabral, S., Lazzarini, S. G., \& de Azevedo, P. F. (2010). Private operation with public supervision: evidence of hybrid modes of governance in prisons. Public Choice, 145(1-2), 281-293

Cassiman, B., \& Veugelers, R. (2006). In search of complementarity in innovation strategy: internal R\&D and external knowledge acquisition. Management science, 52(1), 68-82. 
Conde, M. V. F., \& Araújo-Jorge, T. C. D. (2003). Innovation models and conceptions: transition of paradigm, the Brazilian S\&T reform and conceptions of managers from a public health research institution. Ciência \& Saúde Coletiva, 8(3), 727-741.

Dyer, J. H., \& Singh, H. (1998). The relational view: cooperative strategy and sources of interorganizational competitive advantage. Academy of management review, 23(4), 660-679.

Gebauer, H., Worch, H., \& Truffer, B. (2012). Absorptive capacity, learning processes and combinative capabilities as determinants of strategic innovation. European Management Journal, 30(1), 57-73.

Granovetter, M. (1985). Economic action and social structure: the problem of embeddedness. American journal of sociology, 481-510. Horwitz, S. K. (2005). The compositional impact of team diversity on performance: Theoretical considerations. Human resource development review, 4(2), 219-245.

Hoskisson, R. E., Hitt, M. A., \& Hill, C. W. (1993). Managerial incentives and investment in R\&D in large multiproduct firms. Organization Science, 4(2), 325-341.

Kirch, G., Lima, J. B. N. D., \& Terra, P. R. S. (2012). Determinantes da defasagem na divulgação das demonstrações contábeis das companhias abertas brasileiras. Revista Contabilidade \& Finanças, 23(60), 173-186.

Kurtzberg, T. R. (2005). Feeling creative, being creative: An empirical study of diversity and creativity in teams. Creativity Research Journal, 17(1), 51-65.

Klein, P. G., Mahoney, J. T., McGahan, A. M., \& Pitelis, C. N. (2013). Capabilities and strategic entrepreneurship in public organizations. Strategic Entrepreneurship Journal, 7(1), 70-91.

Mapa- Ministério da Agricultura Pecuária e Abastecimento- Assessoria de Gestão Estratégica. (2011). Projeções do Agronegócio Brasil 2010/11 a 2020/21.2011. http://www.agricultura.gov.br/arq_editor/file/Ministerio/gestao/projecao/PROJECOES20 Soybean - A Review / Book 3\%20DO\%20AGRONEGOCIO\%202010-11\%20a\%202020-21\%20-\%202_0.pdf, acessado em 02 fev 2013.

McCullagh, P., \& Nelder, J. A. (1989). Generalized linear models. London: Chapman and Hall

Porter, M. E. (1996). What is strategy?. Published November.

Puente-Palacios, K. E., Seidl, J., \& Silva, R. A. D. D. (2008). Ser ou parecer diferente: o papel da diversidade na satisfação de equipes de trabalho.Revista Psicologia, 8(2), 79-97.

Rezaie, R., McGahan, A. M., Daar, A. S., \& Singer, P. A. (2012). Innovative drugs and vaccines in China, India and Brazil. Nature Biotechnology, 30(10), 923-926.

Roper, S., Vahter, P., \& Love, J. H. (2013). Externalities of openness in innovation. Research Policy, 42(9), 1544-1554. 
Salles Filho, S. (2000). Ciência, tecnologia e inovação. In Ciência, tecnologia e inovação. Editorial Komedi.

Salles Filho, S., \& Bonacelli, M. B. (2005). Trajetórias e agendas para os institutos e centros de pesquisa no Brasil. Revista Parcerias Estratégicas, (20).

Schumpeter, J. A. (1982). Teoria do desenvolvimento econômico: uma investigação sobre lucros, capital, crédito, juro eo ciclo econômico. Abril Cultural.

Roberts, J. (2010). Designing incentives in organizations. Journal of institutional economics, 6(01), 125-132.

Spers, E. E., Zylbersztajn, D., \& Lazzarini, S. (2003). Percepção do consumidor sobre os mecanismos de qualidade e segurança em alimentos. Revista de Administração da Unimep, 1(1), 57-80.

Teece, D. J. (2010). Business models, business strategy and innovation. Long Range Planning, 43(2), $172-194$

Teece, D., \& Pisano, G. (1994). The dynamic capabilities of firms: an introduction. Industrial and corporate change, 3(3), 537-556.

Terziovski, M. (2010). Innovation practice and its performance implications in small and medium enterprises (SMEs) in the manufacturing sector: a resource-based view. Strategic Management Journal, 31(8), 892-902.

Van Knippenberg, D., \& Schippers, M. C. (2007). Work group diversity. Annu. Rev. Psychol., 58, 515-541.

Uzzi, B. (1997). Social structure and competition in interfirm networks: The paradox of embeddedness. Administrative science quarterly, 35-67.

Williamson, O.E. (1999). Public and private bureaucracies: a transaction cost economics perspective. Journal of Law, Economics and Organization 15 (1):306-342

Zaheer, A., Gulati, R., \& Nohria, N. (2000). Strategic networks. Strategic management journal, 21(3), 203.

Zawislak, P. A., Cherubini Alves, A., Tello-Gamarra, J., Barbieux, D., \& Reichert, F. M. (2012). Innovation capability: From technology development to transaction capability. Journal of technology management \& innovation, 7(2), 14-27. 


\title{
Using strategy lenses to understand the performance determinants in Agricultural research projects
}

\begin{abstract}
This wok aims to identify the factors affecting the performance of agricultural research and innovation projects in a public organization (EMBRAPA). By using a sample of 40 projects, the paper shows that heterogeneous teams, partnerships, alliances and networks present a positive impact on the number of technologies generated by the each research project. Results also suggest a trade-off between scientific papers and the number of technologies. The number of papers is correlated to team homogeneity and to the number of researchers involved in the projects.
\end{abstract}

Key words: Strategy; Agricultural research and innovation; Performance.

Data do recebimento do artigo: 05/08/2014

Data do aceite de publicação: 11/11/2015

\footnotetext{
${ }^{\text {i }}$ Recursos Extra-tesouro são recursos provenientes de outras fontes financiadoras, cuja execução não está sujeita ao arcabouço burocrático da Lei 8.666/93, que disciplina a utilização do recurso do Tesouro-Nacional, fonte principal de financiamento da Embrapa.

ii A Embrapa adota em seu sistema de gestão portfólios de projetos denominados macroprogramas, os quais são orientados para a gestão de carteiras de projetos e processos.
}

Revista de Administração e Inovação, São Paulo, v. 12, n.4 p. 119-139, out./dez. 2015. 